\title{
A Model for Anisotropic Epitaxial Lateral Overgrowth
}

\author{
M. Khenner ${ }^{1}$ R.J. Braun ${ }^{1}$ and M.G. Mauk $^{2}$ \\ ${ }^{1}$ Department of Math. Sciences \\ University of Delaware, Newark, DE 19716
}

2 AstroPower, Inc.

Solar Park, Newark, DE 19716

February 28, 2002

\begin{abstract}
The model for anisotropic crystal growth on a substrate covered by a mask material with a periodic series of parallel long trenches where the substrate is exposed to the vapor phase is developed. The model assumes that surface diffusion and deposition flux are the main mechanisms of the growth, and that the three key surface quantities (energy, mobility and adatom diffusivity) are anisotropic with either four- or six-fold symmetry. A geometrical approach to the motion of crystal surface in two dimensions is adopted and nonlinear evolution equations are solved by a finite-difference method. The model allows the direct computation of the crystal surface shape and the study of effects due to finite mask thickness. As in experiments, lateral overgrowth of crystal onto the mask is found, as well as comparable crystal shapes; the anisotropy of the surface mobility is found to play the dominant role in the shape selection. The amount of the overgrowth and the shapes can be effectively controlled by orienting the fast and slow growth directions with respect to the substrate.
\end{abstract}

Keywords: selective epitaxy, vapor phase epitaxy, epitaxial lateral overgrowth, computer simulation.

\section{Introduction}

In this paper, a computational model of the crystal growth on a patterned masked substrate is developed. When the substrate is partially masked, the epitaxial crystals may be useful as electronic devices as grown or for process diagnostics $[1,2]$. In the growth process we model here, the crystal to be grown is deposited on a substrate which is exposed to the vapor through etched windows, or trenches, in a dielectric mask (e.g., a deposited $\mathrm{SiO}_{2}$ film). When the mask and trench widths are small $(<10 \mu \mathrm{m})$, the vapor phase transport can be neglected and the surface diffusion is appreciable; this has been demonstrated experimentally [1]-[5]. 
Following [1], the growth process in these small geometries, which may allow the crystal to extend over the mask is referred to as epitaxial lateral overgrowth (ELOG). Enhanced growth ("bump") may be observed at the edge of the crystal growing over the mask where the thickness is increased relative to the thickness in the center of the trench $[4,6]$. Nucleation of the crystal phase on the mask may occur under some conditions [6]-[9]; we ignore nucleation on the mask in our modeling.

If field variables around the crystal surface, such as the elastic stresses in the growing crystal, are neglected, then we can develop a "geometric model" of the crystal growth [10, 11]. Geometric models of crystal growth have played an important role in the theory of solidification; for a review, see [10]. In growth from an undercooled melt, such theories were the first to elucidate the role of anisotropy in reducing and eliminating tip splitting. Geometric models have also been useful in studying less unstable growth processes; some recent examples include $[12,13,14]$. In these works, epitaxial growth of a crystal on the flat surface of a substrate is studied and issues such as the saturation and coarsening of the mound structure are investigated. In our case, the mask introduces a length scale which raises new questions. Growth on a substrate with complex patterns has been studied in $[15,16,17]$; that work also includes re-emission of atoms from the crystal surface and other effects.

This paper is a continuation of our work on modeling ELOG in a regime in which transport in the vapor can be neglected. A model with isotropic surface properties was developed in [18]; those properties included surface energy, surface mobility, and adatom diffusivity. In the model, the normal velocity at any point on the crystal surface is a function of the curvature and its derivatives; marker points on the surface are advanced using parametric evolution equations for those points which use the normal velocity function. Every advancing step is followed by remeshing to have an even spacing between markers. The details of the model and numerical algorithm appear in [18]; the latter is similar to that of Wong et al. [19] In this work, we demonstrate that the dynamic evolution of the concentration of adatoms on the mask may be well approximated by using a constant surface diffusion flux at the contact point between the crystal and the mask. The comparison is given in an appendix; the constant flux model is used throughout the computation presented here. The model does not assume that there is or is not lateral overgrowth a priori; the overgrowth occurs naturally during the computation.

With the isotropic model, some qualitative agreement with experiment was observed. As the crystal grows onto the mask, a bump may form near the contact point; it is subsequently smoothed by diffusion on the crystal surface. The relative extent of the overgrowth onto the mask and the height of the crystal at the middle of the window show qualitative agreement with experiment. The isotropic model did not reflect the fact the most electronic materials are anisotropic. Grown crystals certainly show visible signs of anisotropy in their shapes, which may have sharp corners or facets.

In this paper, we generalize the surface properties of our previous model to be anisotropic (though our current formulation does not allow for the anisotropies to be large enough to cause corners or facets). The surface diffusion, energy and mobility may all have four- or six-fold symmetry. We find that these anisotropic properties can greatly exaggerate the formation of distortions or bumps at the crystal edge, and the resulting shapes show striking 
resemblance to some grown crystals reported in the literature. We believe that our results suggest possible ways to grow useful shapes for device development by orienting the crystal with respect to the substrate and mask surfaces. We also aim to provide a more comprehensive framework with which to view the choices for orienting the crystal and what the likely outcome during growth from a straight via will be.

The outline of the paper is as follows. In section 2, we formulate the anisotropic model; section 3 gives numerical results. Section 4 contains the discussion and conclusions, where we describe in more detail the comparison with experiment and the limitations of the model. In

an appendix, we demonstrate that using the constant flux condition at the edge of the growing crystal is a good approximation to the problem with the time dependent concentration profile on the mask.

\section{Anisotropic model}

Fig. 1 shows a sketch of the mathematical situation; we examine the growth behavior on a partial cross-section which is a line segment extending from the center line of one mask surface at $x^{\prime}=-\ell$ to the center of the adjacent trench at $x^{\prime}=L$. We assume the surface behavior is constant in the perpendicular direction to legitimize this two-dimensional study.

\subsection{Surface diffusion over the mask}

The interval $-\ell \leq x^{\prime} \leq 0$ corresponds to the masked area of the substrate. Here we study the surface diffusion of the concentration $n_{m}=n_{m}\left(x^{\prime}, t^{\prime}\right)$ of adatoms on the horizontal part of the mask not yet covered by the growing crystal:

$$
\frac{\partial n_{m}}{\partial t^{\prime}}=D_{s}^{(m)} \frac{\partial^{2} n_{m}}{\partial\left(x^{\prime}\right)^{2}}+J_{g}-\frac{n_{m}}{\tau_{m}}, \quad-\ell \leq x^{\prime} \leq x_{*}^{\prime}\left(t^{\prime}\right)
$$

$J_{g}$ represents uniform flux of atoms from a vapor impinging on the mask. The term $n_{m} / \tau_{m}$ provides the concentration of atoms desorbing from the surface back into the vapor. The constant $\tau_{m}$ is the mean adatom residence time on the mask. Finally, $D_{s}^{(m)}$ is the surface diffusivity of adatoms on the mask. (2.1) has been given in [20,21], for example.

Appended to (2.1) is a symmetry boundary condition,

$$
\left.\frac{\partial n_{m}}{\partial x^{\prime}}\right|_{\left(-\ell, t^{\prime}\right)}=0 .
$$

We assume that the adatoms close to the crystal will be absorbed quickly; thus we take the boundary condition at the contact point to be a perfect sink:

$$
n_{m}\left(x_{*}^{\prime}\left(t^{\prime}\right), t^{\prime}\right)=0 \text {. }
$$

An initial condition is also needed to define this part of the problem completely, say

$$
n_{m}\left(x^{\prime}, 0\right)=0, \quad-\ell \leq x^{\prime} \leq 0 .
$$




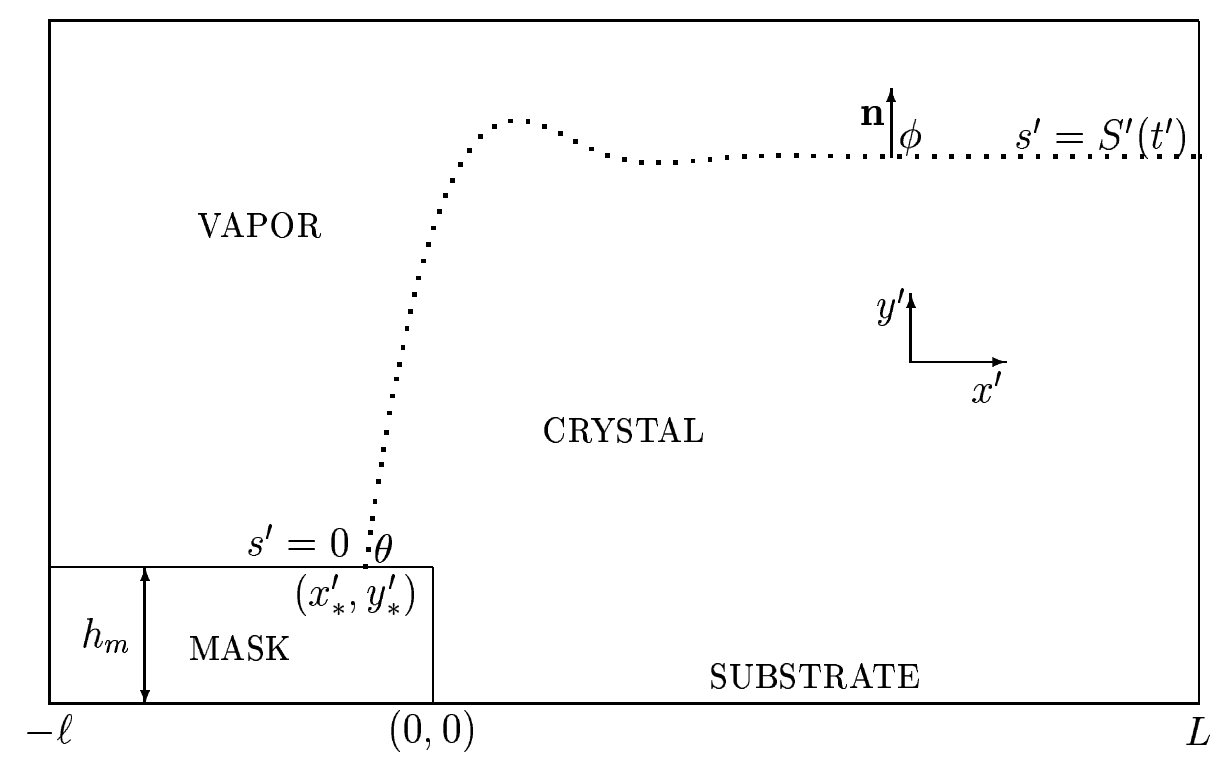

Figure 1: A sketch of the mathematical situation. The free surface of the growing crystal (curve in two dimensions) is defined parametrically as $y^{\prime}=y^{\prime}\left(s^{\prime}, t^{\prime}\right), x^{\prime}=x^{\prime}\left(s^{\prime}, t^{\prime}\right), 0 \leq s^{\prime} \leq S^{\prime}\left(t^{\prime}\right)$, where $s^{\prime}$ is the arc length along the curve and $S^{\prime}$ is the total arc length of the curve (primes denote dimensional variables). The surface is sketched such that a crystal overgrowth onto the mask and the region of the enhanced growth $[4,6]$ (bump) near the contact point $\left(x_{*}^{\prime}\left(t^{\prime}\right), y_{*}^{\prime}\left(t^{\prime}\right)\right)$ are shown. $\phi$ is the angle that the unit normal, $\mathbf{n}$, makes with the horizontal axis; $\mathbf{n}$ points into the vapor phase.

To summarize, there exist two options for every atom arriving to the top, horizontal part of the mask at $-\ell \leq x^{\prime} \leq 0$ : it can either desorb back into the vapor phase or be transported by surface diffusion toward the growing crystal. The diffusing adatoms are incorporated in the crystal at the contact point $\left(x_{*}^{\prime}\left(t^{\prime}\right), y_{*}^{\prime}\left(t^{\prime}\right)\right)$ as they impinge on the crystal. At the initial stage of the growth the adatoms need to diffuse over the mask corner and down the vertical sidewall of the trench prior to incorporation into the crystal. Here we ignore surface diffusion along the vertical part of the mask and instead assume that all adatoms reaching the corner of the mask are incorporated into the growing crystal instantly. This approximation is good if the mask thickness is much less than the mask width, that is if $h_{m} \ll \ell$. In ELOG the ratio $h_{m} / \ell$ is usually $\ll 0.1$ [2]-[4],[6]-[9]. In all our simulations $h_{m} / \ell=0.1$ (ref. Table 1 ). However, in an appendix we show the solution of the full diffusion problem on the mask.

Both the top and the vertical sidewall of the mask are assumed to be physically equivalent. As a particular consequence, the equilibrium contact angle $\theta\left(\gamma, \gamma_{m}\right)$ which the crystal surface forms with the mask at the contact point (as a result of the assumed thermodynamic equilibrium in the vicinity of the junction [22]) should be the same constant value on either side of the mask corner, ref. Fig. 1. Here $\gamma$ and $\gamma_{m}$ are the crystal-vapor and crystal-mask surface energies, respectively, and $\theta$ is thus a material parameter. 


\subsection{Model for the crystal surface}

To account for anisotropic evolution of the crystal surface, we assume the dependence of the mobility, $M$, surface diffusivity, $D_{s}^{(c)}$, and surface energy, $\gamma$, on the angle of the unit normal, $\phi$, to the surface:

$$
M=M_{0} \hat{M}(\phi), \quad D_{s}^{(c)}=D_{0} \hat{D}(\phi), \quad \gamma=\gamma_{0} \hat{\gamma}(\phi),
$$

where $M_{0}, D_{0}, \gamma_{0}$ are mean values, and $\hat{M}(\phi), \hat{D}(\phi)$ and $\hat{\gamma}(\phi)$ are given by

$$
\begin{gathered}
\hat{M}(\phi)=1+\epsilon_{m} \cos \left[p\left(\phi+\beta_{m}\right)\right], \\
\hat{D}(\phi)=1+\epsilon_{d} \cos \left[p\left(\phi+\beta_{d}\right)\right], \\
\hat{\gamma}(\phi)=1+\epsilon_{\gamma} \cos \left[p\left(\phi+\beta_{\gamma}\right)\right],
\end{gathered}
$$

where (i) constants $\epsilon_{m}, \epsilon_{d}, \epsilon_{\gamma}$ determine the degree of the anisotropy of $M, D_{s}^{(c)}$ and $\gamma$, respectively, (ii) value of $p$ (4 or 6 ) specifies the type of anisotropy (four- or six-fold), (iii) $\beta_{m}, \beta_{d}$ and $\beta_{\gamma}$ are phase shifts (we will use the phase shifts to control fast and slow growth directions with respect to the horizontal substrate, for example).

As stated in the Introduction, the interface moves via two mechanisms [23]. The first is from a flux of atoms to the interface from the vapor; this contribution to the normal velocity of the interface is proportional to the jump in the chemical potential across the interface, viz.,

$$
M A\left(\mu_{V}^{T}-\mu_{c}\right) .
$$

Here $A=\partial n_{s} / \partial \mu$ is the change of surface concentration $n_{s}$ with chemical potential $\mu ; \mu_{c}$ is chemical potential of the crystal surface and is given by [22]

$$
\mu_{c}=\mu_{\infty}+\Omega\left(\gamma+\frac{\partial^{2} \gamma}{\partial \phi^{2}}\right) \kappa
$$

where $\Omega$ is the atomic volume, $\kappa$ is the curvature of the crystal surface and $\mu_{\infty}$ is the reference value of the chemical potential for the crystal with a flat surface. $\mu_{V}^{T}$ is the chemical potential of the vapor and it may be written as $\mu_{V}^{T}=\mu_{V}+\mu_{\infty}$.

The second contribution is from the surface diffusion of adatoms along the interface; the current of adatoms $J_{s}$ is proportional to the gradient of the chemical potential along the surface. For the curve in the plane, the surface gradient is the derivative with respect to arc length, hence

$$
J_{s}=-A D_{s}^{(c)} \frac{\partial \mu_{c}}{\partial s^{\prime}} .
$$

The contribution to the rate of increase in the atoms per unit area (proportional to the normal velocity) is proportional to the surface divergence of this flux; for the curve, we have $\left(-\partial J_{s} / \partial s^{\prime}\right)$.

The net rate of increase in the number of atoms per unit area (or the time rate of change of the concentration) may be written as $V_{n} / \Omega$, where the $\Omega$ is the atomic volume and $V_{n}$ is the normal velocity. Combining the flux from the vapor and surface diffusion contributions results in

$$
V_{n}=A \Omega^{2} \frac{\partial}{\partial s^{\prime}}\left[D_{s}^{(c)} \frac{\partial}{\partial s^{\prime}}\left\{\left(\gamma+\frac{\partial^{2} \gamma}{\partial \phi^{2}}\right) \kappa\right\}\right]+A \Omega M \mu_{V}-A \Omega^{2} M\left(\gamma+\frac{\partial^{2} \gamma}{\partial \phi^{2}}\right) \kappa .
$$


The mobility parameter $M$ is the proportionality between the speed of a planar interface with a certain orientation and the chemical potential difference between the crystal and the vapor. The mobility has been estimated in the isotropic case by Coriell and Turnbull [24] and in the anisotropic case by Mikheev and Chernov [25]. The surface energy $\gamma$ is the change in the chemical potential with curvature. For some materials, one can look at the shapes of liquid drops in a solid matrix or the shape of solid particles in the melt to determine the surface energy as a function of orientation [26]. In [26], it is stated that only five materials have had their surface energy anisotropy experimentally determined; whatever the number is now, it is clear that this is a difficult measurement. For electronic materials, recent statistical mechanical approaches can use atomic scale imaging to determine the surface energy of some materials and its anisotropy in the neighborhood of a facet [27]. The surface diffusion coefficient is perhaps the hardest to measure; this coefficient models the hopping of the atoms on the crystal surface that redistribute matter there to the continuum shape change that this may cause. The measurement of these coefficients is difficult; one can measure a tracer diffusion coefficient that is for a single atom, or a continuum diffusion coefficient for continuum mass transport [28, 29]. Diffusion coefficients may be measured on a particular surface, and some orientation dependence may be inferred; measuring the orientation dependence is a challenging task. For more detail, see [28, 29]. As always seems to be the case in problems involving interfaces, it is difficult to know the interfacial properties accurately. In this work, we will choose what we believe to be reasonable parameters.

In [18], a geometrical approach to the problem of the motion of an open curve (the crystal surface) was adopted. The dimensional parametric evolution equations (involving the normal velocity) are given in the section 2.2.1 of [18]; the nondimensional forms will be given in the next section. Next, we describe physical boundary and initial conditions which will be used for the computation.

First, we assume the following conditions hold for the contact point location $x_{*}^{\prime}, y_{*}^{\prime}$ (see Fig. 1):

$$
\begin{gathered}
x_{*}^{\prime}=0, \quad \text { if } \quad 0 \leq y_{*}^{\prime}<h_{m}, \\
y_{*}^{\prime}=h_{m}, \quad \text { if } \quad-\ell \leq x_{*}^{\prime}<0 .
\end{gathered}
$$

These conditions place the contact point on the mask.

At $x^{\prime}=L$ we assume the symmetry conditions,

$$
\left.\frac{\partial y^{\prime}}{\partial x^{\prime}}\right|_{\left(L, t^{\prime}\right)}=0 \text { and }\left.\frac{\partial^{3} y^{\prime}}{\partial\left(x^{\prime}\right)^{3}}\right|_{\left(L, t^{\prime}\right)}=0
$$

these conditions prevent any flux of atoms across $x^{\prime}=L$.

Initially, the crystal surface is assumed to be a hyperbolic tangent curve that has the desired contact angle $\theta$ with the mask at the contact point:

$$
y^{\prime}\left(s^{\prime}, 0\right)=y_{0}^{\prime}\left[1-\tanh \left(s^{\prime} \frac{\tan \theta}{y_{0}^{\prime}}\right)\right], x^{\prime}\left(s^{\prime}, 0\right)=s^{\prime}, 0 \leq s^{\prime} \leq L,
$$

if $\theta<90^{\circ}$, and

$$
y^{\prime}\left(s^{\prime}, 0\right)=y_{0}^{\prime} \tanh \left(s^{\prime} \frac{\tan \theta}{y_{0}^{\prime}}\right), x^{\prime}\left(s^{\prime}, 0\right)=s^{\prime}, \quad 0 \leq s^{\prime} \leq L,
$$


if $\theta \geq 90^{\circ}$. In (2.13) and (2.14), $y_{0}^{\prime}$ is a small constant which gives the initial thickness of the crystal at $x^{\prime}=0$ or at $x^{\prime}=L$, respectively.

Since $\theta$ stays constant, for $t^{\prime}>0$ we have

$$
\begin{gathered}
\left.\frac{\partial y^{\prime}}{\partial x^{\prime}}\right|_{\left(x_{*}^{\prime}, y_{*}^{\prime}\right)}=\tan \theta, \quad \text { if } \quad-\ell \leq x_{*}^{\prime}<0 \text { and } y_{*}^{\prime}=h_{m}, \\
\left.\frac{\partial y^{\prime}}{\partial x^{\prime}}\right|_{\left(x_{*}^{\prime}, y_{*}^{\prime}\right)}=\tan \left(\theta-90^{\circ}\right), \quad \text { if } \quad x_{*}^{\prime}=0 \text { and } 0 \leq y_{*}^{\prime}<h_{m} .
\end{gathered}
$$

We also match the surface diffusion flux on the horizontal part of the mask with the flux onto the growing crystal by requiring, at $x^{\prime}=x_{*}^{\prime}$ and $t^{\prime}$, that

$$
D_{s}^{(m)} \frac{\partial n_{m}}{\partial x^{\prime}}=A D_{s}^{(c)} \Omega \frac{\partial}{\partial s^{\prime}}\left\{\left(\gamma+\frac{\partial^{2} \gamma}{\partial \phi^{2}}\right) \kappa\right\}
$$

The bounds for $\epsilon_{m}, \epsilon_{d}$ and $\epsilon_{\gamma}$ in (2.6) could be obtained by observing that $M, D_{s}^{(c)}$ and $\gamma+\partial^{2} \gamma / \partial \phi^{2}$ are nonnegative; thus,

$$
0 \leq \epsilon_{m}, \epsilon_{d} \leq 1, \quad 0 \leq \epsilon_{\gamma} \leq \frac{1}{p^{2}-1}
$$

In this paper, we do not study the case $\gamma+\partial^{2} \gamma / \partial \phi^{2}<0$ that results in missing crystallographic orientations [22,30]. Note that the isotropic formulas (2.8) and (2.13) of [18] are recovered from (2.10) and (2.16), respectively, if in (2.6) $\epsilon_{m}=\epsilon_{d}=\epsilon_{\gamma}=0$.

\subsection{Physical parameters and nondimensional evolution problem}

The physical constants listed in Table 1 are representative for ELOG of GaAs-like material at temperatures near $650^{\circ} \mathrm{C}$.

In [18], we investigated the influence of varying geometrical parameters $L, \ell, h_{m}$ and $\theta$ on the isotropic crystal shapes, lateral overgrowth and growth rates. In this study, we fix the parameters listed in Table 1 at their reference values and investigate the influence of anisotropic quantities $p, \epsilon_{m}, \epsilon_{d}, \epsilon_{\gamma}, \beta_{m}, \beta_{d}$ and $\beta_{\gamma}$.

The model equations (2.1)-(2.4) and (2.10)-(2.16) (as well as parametric evolution equations, ref. [18], section 2.2.1) are nondimensionalised using reasonable physical scales [18]. Table 2 contains estimates of the nondimensional parameters.

The parametric evolution equations then become

$$
\begin{gathered}
\frac{\partial x}{\partial t}=V_{n} \frac{\partial y}{\partial s} \\
\frac{\partial y}{\partial t}=-V_{n} \frac{\partial x}{\partial s} .
\end{gathered}
$$

In (2.18), the nondimensional normal velocity is given by

$$
V_{n}=\epsilon D \frac{\partial}{\partial s}\left[\hat{D}(\phi) \frac{\partial}{\partial s}\left\{\left(\hat{\gamma}(\phi)+\frac{\partial^{2} \hat{\gamma}(\phi)}{\partial \phi^{2}}\right) K\right\}\right]+J \hat{M}(\phi)-\delta\left(\hat{\gamma}(\phi)+\frac{\partial^{2} \hat{\gamma}(\phi)}{\partial \phi^{2}}\right) \hat{M}(\phi) K
$$




\begin{tabular}{|c|c|c|}
\hline Constant & Description & Value/Units \\
\hline \hline$J_{g}$ & Atomic flux from vapor & $10^{15}$ atoms $/\left(\mathrm{cm}^{2} \cdot \mathrm{sec}\right)$ \\
$\tau_{m}$ & Mean residence time of atoms on mask & $1 \mathrm{sec}$ \\
$D_{s}^{(m)}$ & Diffusivity on mask & $5 \times 10^{-8} \mathrm{~cm}^{2} / \mathrm{sec}$ \\
$D_{0}$ & Mean diffusivity on crystal surface & $2 \times 10^{-8} \mathrm{~cm}^{2} / \mathrm{sec}$ \\
$\Omega$ & Atomic Volume & $2 \times 10^{-23} \mathrm{~cm}^{3} /$ atom \\
$\gamma_{0}$ & Mean surface energy & $10^{3} \mathrm{ergs} / \mathrm{cm}^{2}$ \\
$A$ & Change in concentration/Change in chemical potential & $2 \times 10^{28} \mathrm{atoms} /\left(\mathrm{erg}^{2} \mathrm{~cm}^{2}\right)$ \\
$\mu_{V}$ & Chemical potential in the vapor & $3 \times 10^{-13} \mathrm{erg} \cdot \mathrm{atom}$ \\
$M_{0}$ & Mean mobility & $10 \mathrm{sec}-1$ \\
$L$ & Width of substrate region & $5 \times 10^{-4} \mathrm{~cm}(5 \mu \mathrm{m})$ \\
$\ell$ & Width of mask region & $5 \times 10^{-4} \mathrm{~cm}(5 \mu \mathrm{m})$ \\
$h_{m}$ & Mask thickness & $5 \times 10^{-5} \mathrm{~cm}(0.5 \mu \mathrm{m})$ \\
$\theta$ & Contact angle & $45^{\circ} \mathrm{or} 120^{\circ}$ \\
\hline
\end{tabular}

Table 1: Physical parameters.

\begin{tabular}{|c|c|c|}
\hline Constant & Expression & Approximate Value (for $L=\ell=5 \mu \mathrm{m})$ \\
\hline \hline$\epsilon$ & $D_{0} / D_{s}^{(m)}$ & $4 \times 10^{-1}$ \\
$D$ & $\left(\Omega^{2} A \gamma_{0}\right) /\left(L^{2}\right)$ & $3 \times 10^{-8}$ \\
$\delta$ & $\left(M_{0} \Omega^{2} \gamma_{0} A\right) / D_{s}^{(m)}$ & $1.6 \times 10^{-6}$ \\
$\alpha$ & $L^{2} /\left(\tau_{m} D_{s}^{(m)}\right)$ & 5 \\
$J$ & $\left(\Omega A \mu_{V} M_{0} L\right) / D_{s}^{(m)}$ & $1.2 \times 10^{-2}$ \\
$\bar{h}_{m}$ & $h_{m} / L$ & $10^{-1}$ \\
$\beta$ & $\left(A \Omega \gamma_{0}\right) /\left(\tau_{m} J_{g} L\right)$ & $8 \times 10^{-4}$ \\
$d$ & $\ell / L$ & 1 \\
$f_{m}$ & $\sqrt{\alpha} \tanh (\sqrt{\alpha} d)$ & 2.18 \\
\hline
\end{tabular}

Table 2: Nondimensional parameters. 
where $\hat{M}(\phi), \hat{D}(\phi), \hat{\gamma}(\phi)$ are given by $(2.6)$ and

$$
K=\left(\frac{\partial^{2} y}{\partial s^{2}} \frac{\partial x}{\partial s}-\frac{\partial^{2} x}{\partial s^{2}} \frac{\partial y}{\partial s}\right)
$$

is the nondimensional curvature. The angle of the normal, $\phi_{i}$, at the $i$ th grid node on the curve (the latter is characterized by value, $s_{i}$, of the arc length; $i=0 . . N-1$, where $N$ is the number of grid nodes) could be obtained, for example, by integrating curvature, $K(s)$, along the curve:

$$
\phi_{i}=\int_{s_{i}}^{s_{N-1}} K(s) d s .
$$

We now have that $x=1$ corresponds to $s=S(t)=s_{N-1}$ and the contact point $\left(x_{*}, y_{*}\right)$ corresponds to $s=s_{0}=0$, see Fig. 1. The boundary conditions are:

$$
\begin{gathered}
\left.\frac{\partial y}{\partial s}\right|_{(S(t), t)}=0,\left.\quad \frac{\partial^{3} y}{\partial s^{3}}\right|_{(S(t), t)}=0, \\
\left.\frac{\partial y / \partial s}{\partial x / \partial s}\right|_{(0, t)}=\tan \theta, \quad \text { if } \quad-d \leq x_{*}<0 \text { and } y_{*}=\bar{h}_{m}, \\
\left.\frac{\partial y / \partial s}{\partial x / \partial s}\right|_{(0, t)}=\tan \left(\theta-90^{\circ}\right), \quad \text { if } \quad x_{*}=0 \text { and } 0 \leq y_{*}<\bar{h}_{m} .
\end{gathered}
$$

and

$$
\left.\frac{\partial}{\partial s}\left\{\left(\hat{\gamma}(\phi)+\frac{\partial^{2} \hat{\gamma}(\phi)}{\partial \phi^{2}}\right) K\right\}\right|_{(0, t)}=\frac{f_{m}}{\epsilon \beta \hat{D}\left(\phi_{0}\right)} .
$$

In (2.24), $f_{m}$ is a constant surface diffusion flux from mask, which emerges from the (analytical) steady state solution to nondimensionalized initial boundary value problem (2.1)-(2.4) assuming the contact point position is fixed at $x_{*}(t)=0$. When the contact point moves, the same steady flux is not available; however, as it was done in [18], we will assume a constant flux $f_{m}$ from the mask at the contact point for the rest of this paper. In an appendix, we show that this is quite a good approximation for the parameters considered here.

The nondimensional initial conditions are

$$
y(s, 0)=y_{0}\left(1-\tanh \left(s \frac{\tan \theta}{y_{0}}\right)\right), x(s, 0)=s, 0 \leq s \leq 1,
$$

if $\theta<90^{\circ}$, and

$$
y(s, 0)=y_{0} \tanh \left(s \frac{\tan \theta}{y_{0}}\right), x(s, 0)=s, \quad 0 \leq s \leq 1,
$$

if $\theta \geq 90^{\circ}$. Here $y_{0}$ is a constant specifying the initial crystal thickness; these forms give the correct contact angle value at $s=0$.

And, the nondimensional coordinates of the contact point satisfy

$$
x_{*}=0, \quad \text { if } \quad 0 \leq y_{*}<\bar{h}_{m},
$$




$$
y_{*}=\bar{h}_{m}, \quad \text { if } \quad-d \leq x_{*}<0 .
$$

Evolution problem (2.18)-(2.27) is solved by a finite-difference method (namely, the marker particles method) described in [18]; our approach is the variation of the method used in [19] for the study of capillarity driven motion of solid film wedges. The solution yields the surface profile (in the $x y$-plane), dynamically evolving in time.

\section{$3 \quad$ Numerical results}

In this section we present the numerical results for four distinct cases: (i) anisotropic mobility $M$ only $\left(\epsilon_{m} \neq 0, \epsilon_{d}=\epsilon_{\gamma}=0\right.$, ref. (2.5), (2.6)), (ii) anisotropic surface diffusion $D_{s}^{(c)}$ only $\left(\epsilon_{d} \neq 0, \epsilon_{m}=\epsilon_{\gamma}=0\right)$, (iii) anisotropic surface energy $\gamma$ only $\left(\epsilon_{\gamma} \neq 0, \epsilon_{m}=\epsilon_{d}=0\right)$, (iv) anisotropic mobility, surface diffusion and surface energy $\left(\epsilon_{m} \neq 0, \epsilon_{d} \neq 0, \epsilon_{\gamma} \neq 0\right)$. We investigate each case (i)-(iv) separately, trying to emphasize differences in resulting crystal surface shapes and in growth rates in vertical (perpendicular to the substrate) and lateral (onto the mask) directions. Due to the large number of parameters involved in the computation, we consider the physical parameters listed in Table 1, and the resulting nondimensional parameters listed in Table 2 as constants; the values of constants are listed in the third columns of the Tables. The only quantities subject to change are $p, \epsilon_{m}, \epsilon_{d}, \epsilon_{\gamma}, \beta_{m}, \beta_{d}$ and $\beta_{\gamma}$.

The nondimensional problem is posed on the horizontal interval $-1 \leq x \leq 1$ since widths of substrate and masked regions, $L$ and $\ell$ respectively, are equal.

Most of our results (except those presented in Fig. 4) are for the single value of the contact angle, $\theta=120^{\circ}$, since we found (by means of intensive numerical experiments) that the crystal surface shapes far enough away from the contact point are not strongly affected by the contact angle.

For ease of comparison, and unless marked differently in the figure, the last (upper) surface profile at all figures corresponds to the value of nondimensional time $t_{f}=64$. This value was selected only because by that time the numerical results exhibit sufficient amount of the overgrowth onto the mask. The grid resolution $N=500$ marker particles was found to be sufficient in most cases (when necessary, the results were verified by increasing the resolution to $N=1200$ ). The profiles are plotted every 32000 time steps, or at time increments of 4 (unless marked differently in the text or in the figure caption). 
(a)

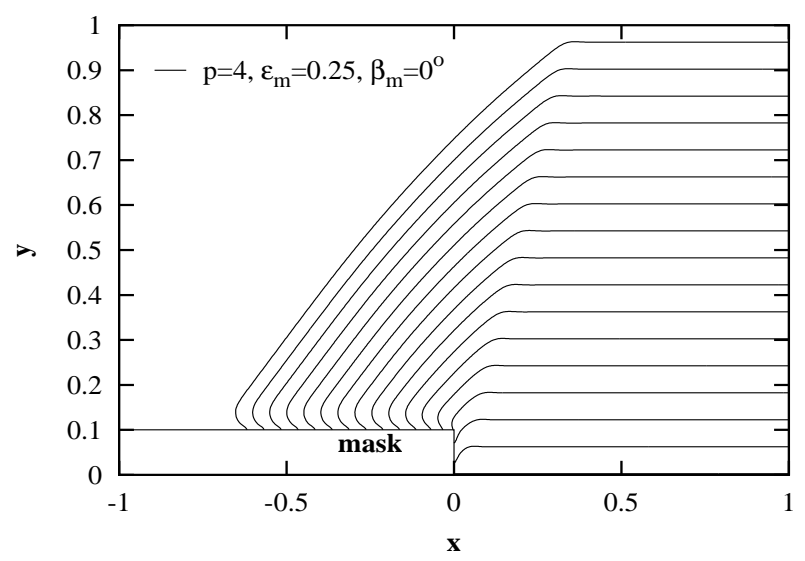

(b)

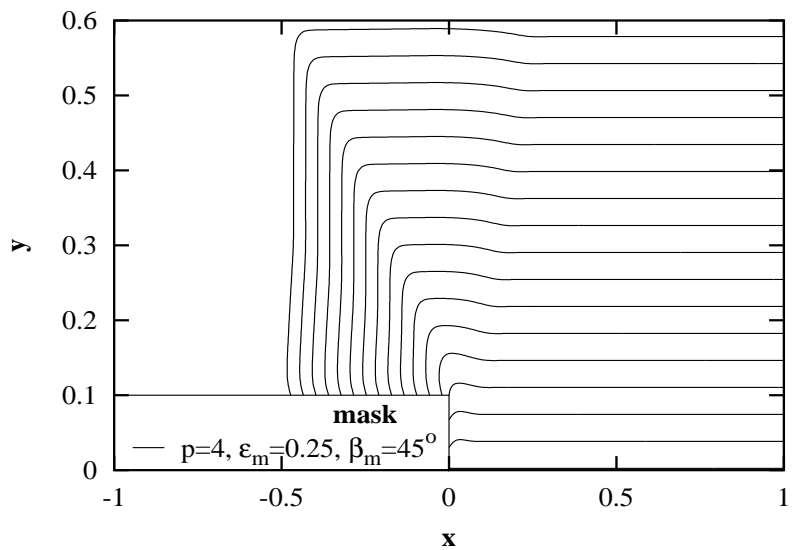

(c)

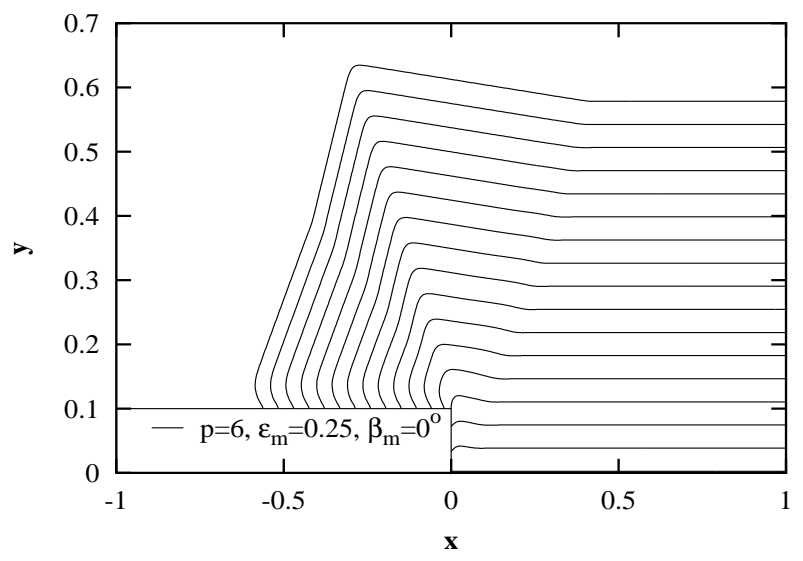

(d)

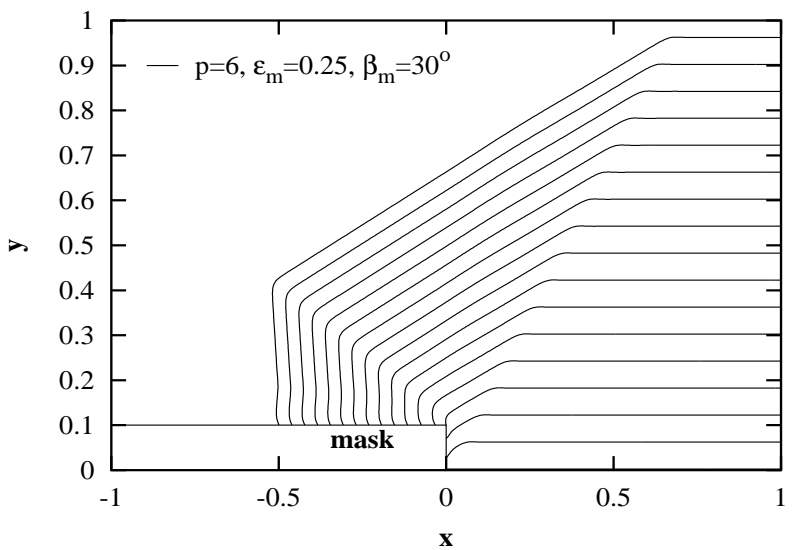

Figure 2: Crystal growth with weakly anisotropic mobility, $M\left(\epsilon_{m}=0.25, \epsilon_{d}=\epsilon_{\gamma}=0\right)$. (a) Four-fold anisotropy $(p=4), \beta_{m}=0^{\circ}$. (b) Same as (a), but $\beta_{m}=45^{\circ}$. (c) Six-fold anisotropy $(p=6), \beta_{m}=0^{\circ}$. (d) Same as (c), but $\beta_{m}=30^{\circ}$. Also note the different scales along $x$ and $y$-axis at this and at other figures in section 3 .

\subsection{Anisotropic mobility $\left(\epsilon_{m} \neq 0\right)$}

Figures $2-4$ show the results of the modeling in the case of anisotropic mobility, $M$. This is where we obtain a fascinating variety of crystal structures, among those many were observed in experimental works on ELOG $[6,8]$. And, since the normal velocity (2.19) is simple in $\hat{M}$, most results yield a clear interpretation. As will be seen, the choice of different values for the phase shift, $\beta_{m}$, in (2.6) allows one to control fast and slow growth directions with respect to the horizontal substrate.

\subsubsection{Weak anisotropy of $M$}

In Fig. 2 we plot crystal surface profiles for a relatively small degree of anisotropy, $\epsilon_{m}=0.25$ (ref. (2.17)). Figures 2(a,b) give results for the four-fold anisotropy case, $p=4$.

When the phase shift is zero, $\beta_{m}=0^{\circ}$ (Fig. 2(a)), we observe the formation and development of truncated-pyramid-like shape [8]. According to (2.5), (2.6) with $\beta_{m}=0^{\circ}$, the 
mobility is maximal in the direction normal to the substrate (at $\phi=90^{\circ}$ ), that is at the horizontal, nonperturbed region of the curve. Since the curvature, $K$, is zero there, then the last term in (2.19) is also zero, and the maximal $M$ at $\phi=90^{\circ}$ yields maximal $V_{n}$. Therefore, the growth is fast in this direction, with the nondimensional crystal thickness reaching $\approx 0.96$ at the final time. Also, the maximal mobility in the lateral direction $\left(\right.$ at $\left.\phi=180^{\circ}\right)$ causes the enhanced overgrowth onto the mask.

It follows from (2.5), (2.6) that the phase shift $\beta_{m}=45^{\circ}$ yields a minimum of $M$ in the direction normal to the substrate, but it supports the growth at the normal angle $\phi=135^{\circ}$. This results in the (imperfect) block-like structure [8] shown in Fig. 2(b), with $90^{\circ}$ and $180^{\circ}$ surfaces.

Figures 2(c,d) give results for the six-fold anisotropy case, $p=6$. The situation here is in a sense opposite to the discussed $p=4$ case. Zero phase shift yields the slow growth in the direction normal to the substrate, and enhanced growth in the directions $\phi=120^{\circ}$ and $\phi=180^{\circ}$ (Fig. 2(c)) [6]. Having $\beta_{m}=30^{\circ}$ results in the nearly faceted crystal shown in Fig. 2(d), with distinct $90^{\circ}, 120^{\circ}$ and $180^{\circ}$ surfaces [8]. The growth is fast in the vertical direction and in the direction $\phi=150^{\circ}$.

\subsubsection{Strong anisotropy of $M$}

In Fig. 3 we plot crystal surface profiles for an almost maximal possible degree of anisotropy, $\epsilon_{m}=0.95$.

In the four-fold case, a very different growth is observed in the case $\beta_{m}=0^{\circ}$ (Fig. $3(\mathrm{a})$ ) and in the case $\beta_{m}=45^{\circ}$ (Fig. 3(b)). If $\beta_{m}=0^{\circ}$, we still observe the truncated pyramid as in the corresponding weakly anisotropic case (compare to Fig. 2(a)), but the growth is confined to the substrate only - the contact point moves so slowly that there is no overgrowth on the mask at all! Instead, the crystal grows very fast vertically, in the center of the substrate region. Also note the perfect shape of this pyramid. The opposite situation is shown in Fig. 3(b), where the crystal growth in the vertical direction is very slow, and the crystal "spreads" along the mask. To complement this figure, we provided the surface profile at $t_{f}=100$ (dashed line) and also the profile which results at $t_{f}=64$ when both the anisotropic mobility and anisotropic surface diffusion, $D_{s}^{(c)}$, are activated (dotted line). The anisotropic surface diffusion further enhances the spreading on the mask. 
(a)

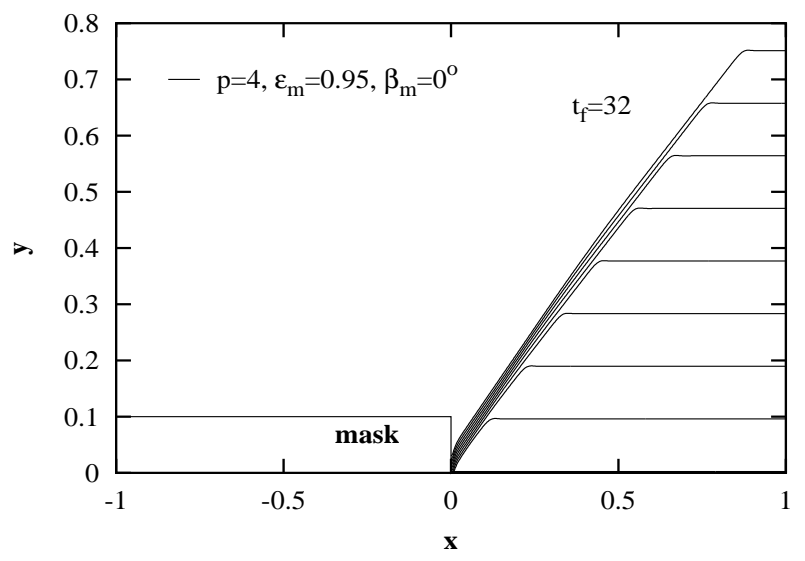

(b)

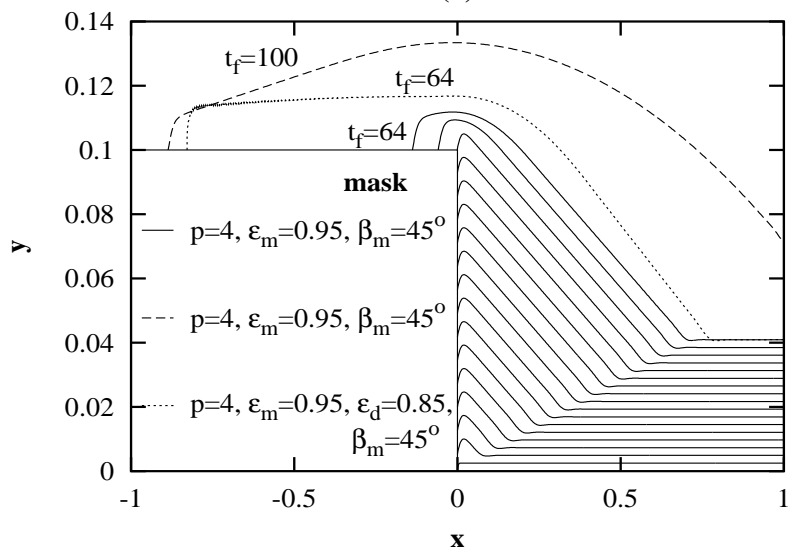

(c)

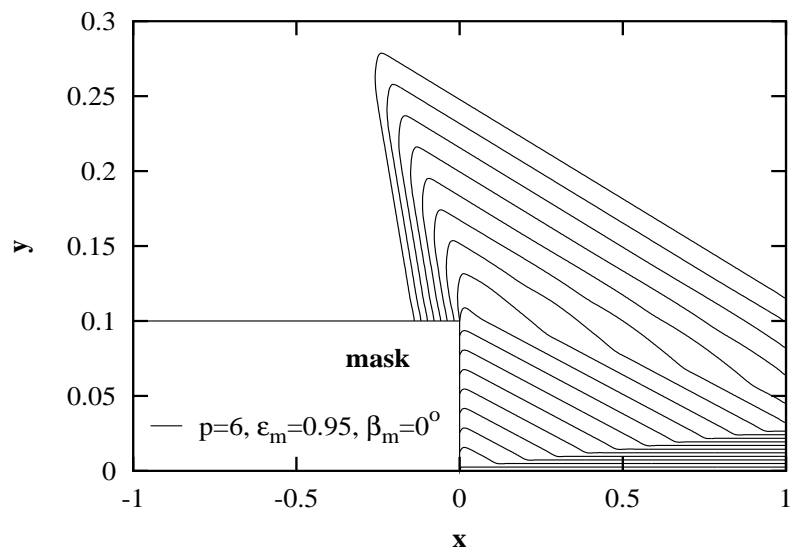

(d)

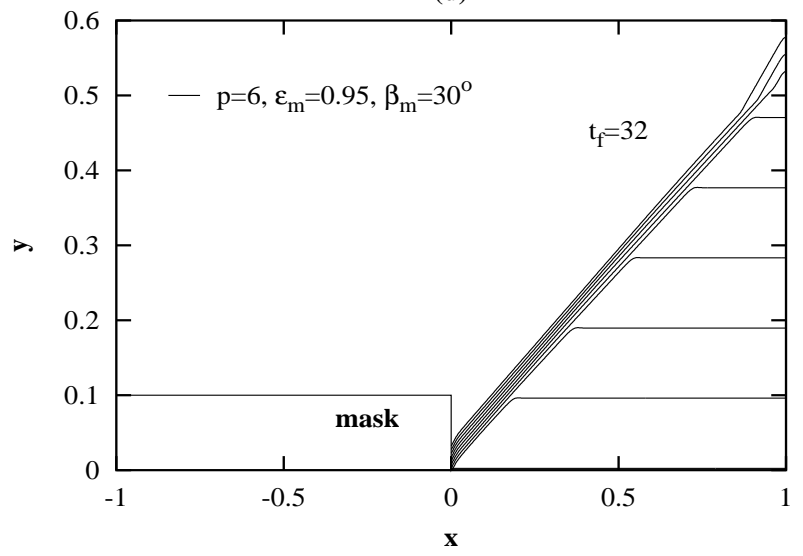

Figure 3: Crystal growth with strong anisotropic mobility, $M\left(\epsilon_{m}=0.95, \epsilon_{d}=\epsilon_{\gamma}=0\right)$. (a) Four-fold anisotropy $(p=4), \beta_{m}=0^{\circ}$. (b) Same as (a), but $\beta_{m}=45^{\circ}$. (c) Six-fold anisotropy $(p=6), \beta_{m}=0^{\circ}$. (d) Same as (c), but $\beta_{m}=30^{\circ}$.

In the six-fold case with $\beta_{m}=0^{\circ}$ (Fig. $3(\mathrm{c})$ ), the crystal initially grows in a fashion which resembles the growth in the previous case $p=4, \beta_{m}=45^{\circ}$ (Fig. 3(b)) but, once the contact point reaches the mask corner, the fastest growth direction become $\phi=120^{\circ}$, as in the case of Fig. 2(c). However, the direction $\phi=180^{\circ}$ is not as fast as in Fig. 2(c), and the overgrowth is relatively small. Having phase shift $30^{\circ}$ (Fig. 3(d)) results in the pyramidal structure similar to one shown in Fig. 3(a), with the growth entirely confined to the substrate. The growth of the $120^{\circ}$ side is, however, faster than in the case of Fig. 3(a), thus resulting in the shrinking of the top surface to zero before the run was terminated. The last three profiles in Fig. 3(d) demonstrate what happens with the computed surface after this event; the pyramid develops a steeper top which eventually takes over the grown shape. Of course, a similar "fate" is expected sooner or later for the crystal surface plotted in Figures 2(a), 2(d), 3(a) near $x=1$. No complex shapes involving sidebranching or tip splitting were observed in our computations.

For the sake of completeness, in Figures $4(\mathrm{a}, \mathrm{b})$ we plot some computed profiles for the contact angle $\theta=45^{\circ}$. These two figures should be compared with Figures 2(c) and 3(c), respectively (for which $\theta=120^{\circ}$ ). Clearly, Figures $4(\mathrm{a}, \mathrm{b})$ represent same types of crystal 
growth as Figures 2(c) and 3(c), although there are substantial differences in the profiles near the contact point. As stated in the beginning of section 3 , this observation is generic for all numerical experiments we conducted.

(a)

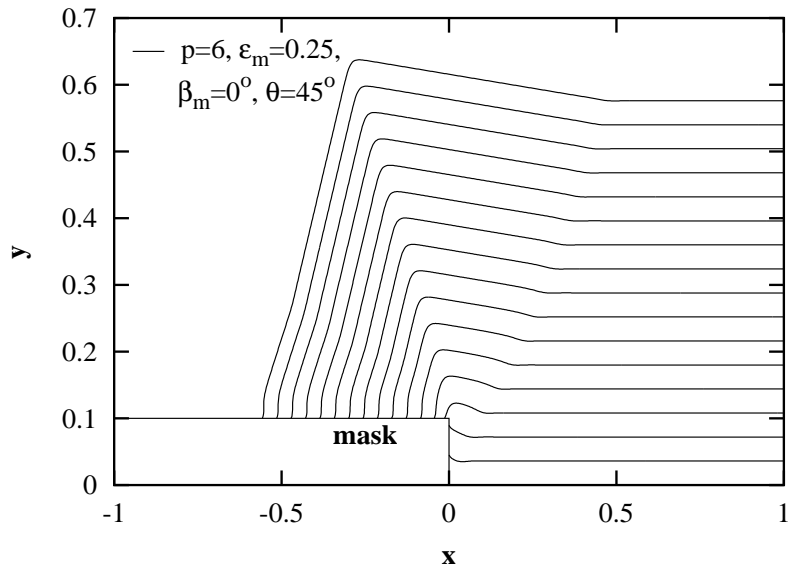

(b)

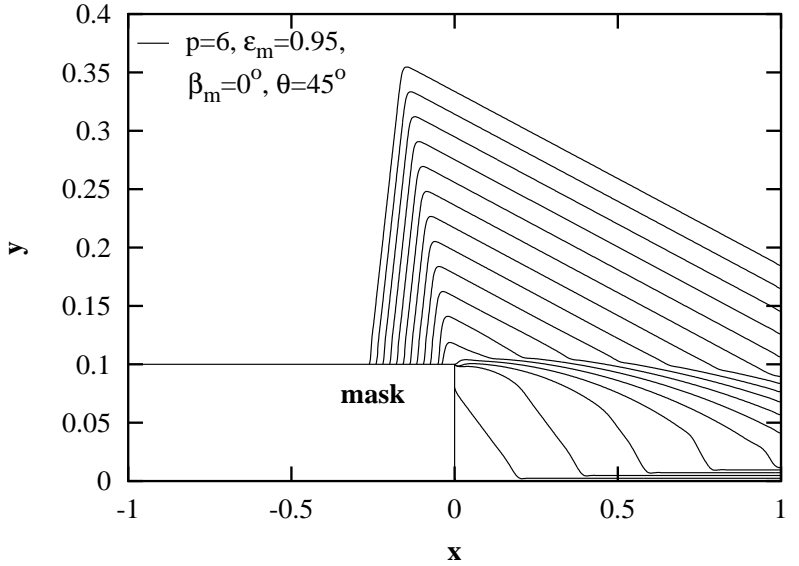

Figure 4: Crystal growth with the six-fold anisotropic mobility, $M$ and with the imposed contact angle $\theta=45^{\circ} ; \epsilon_{d}=\epsilon_{\gamma}=0$. (a) Weak anisotropy; $\epsilon_{m}=0.25$. (b) Strong anisotropy; $\epsilon_{m}=0.95$.

\subsection{Anisotropic surface diffusion $\left(\epsilon_{d} \neq 0\right)$}

Fig. 5 compares the surface profiles for $\epsilon_{d}=0.85$ and $p=4,6$ to isotropic profiles $(p=0)$. In the case of four-fold anisotropy, we plotted profiles for $\beta_{d}=0^{\circ}$ and $\beta_{d}=45^{\circ}$, and in the case of six-fold anisotropy, we plotted profiles for $\beta_{d}=0^{\circ}$ and $\beta_{d}=30^{\circ}$.

For $p=4$, the maximum of $D_{s}^{(c)}$ at $\phi=90^{\circ}$ (e.g., in the unperturbed region close to the center of the substrate) corresponds to $\beta_{d}=0^{\circ}$, but the minimum of $D_{s}^{(c)}$ at $\phi=90^{\circ}$ corresponds to $\beta_{d}=45^{\circ}$, ref. (2.5) and (2.6). Certainly, values of $D_{s}^{(c)}$ at other points on the curve, characterized by $\phi \neq 90^{\circ}$ are also affected by the choice of $\beta_{d}$. Alternatively, for $p=6$ the maximum of $D_{s}^{(c)}$ at $\phi=90^{\circ}$ corresponds to $\beta_{d}=30^{\circ}$, but the minimum of $D_{s}^{(c)}$ at $\phi=90^{\circ}$ corresponds to $\beta_{d}=0^{\circ}$. Thus, the choice of different values for $\beta_{d}$ allows us to study how the shapes of the surface and the growth rates are affected by different values of the surface diffusion coefficient at various parts of the surface, having at the same time fixed degree of anisotropy, $\epsilon_{d}$. We observe, however, that the anisotropy of the surface diffusion alone has very little effect on the surface shape no matter what $\beta_{d}$ is, even though the degree of anisotropy is high $\left(\epsilon_{d}=0.85\right)$; the small difference in shapes occurs near the contact point, and it gradually decreases with the arc length. The former statement may seem in contradiction with the conclusions made in section 3.1.2, where we found that anisotropic surface diffusion enhances the spreading on the mask; however, the difference it makes near the contact point appears to be enough to support the overgrowth in the case of Fig. 3(b).

\subsection{Anisotropic surface energy $\left(\epsilon_{\gamma} \neq 0\right)$}

Fig. 6 compares anisotropic surface profiles to isotropic results. 
(a)

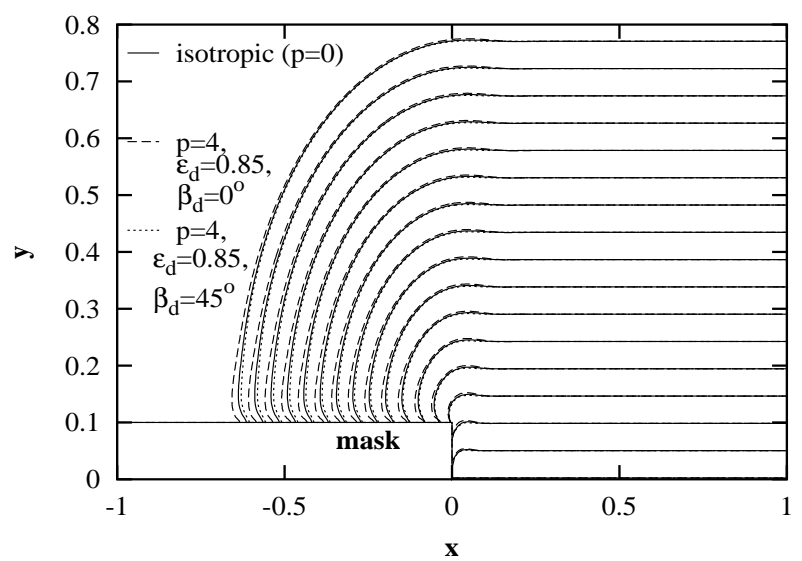

(b)

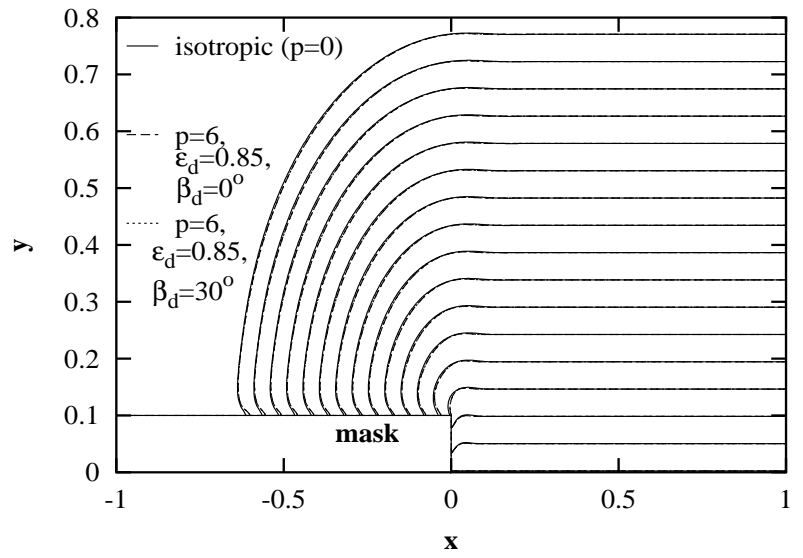

Figure 5: Comparison of anisotropic crystal growth to the isotropic case; the growth anisotropy is due only to the anisotropy of the surface diffusion coefficient, $D_{s}^{(c)}$. (a): $p=4$. (b): $p=6$. For both cases, $\epsilon_{d}=0.85, \epsilon_{m}=\epsilon_{\gamma}=0$.

In the case of four-fold anisotropy ( $p=4$, ref. Fig. 6(a)) we plotted the anisotropic profiles for $\epsilon_{\gamma}=1 / 18$ and $\beta_{\gamma}=0^{\circ}$. The maximum of $\hat{\gamma}$ is in the direction normal to the substrate (at $\phi=90^{\circ}$ ) when $\beta_{\gamma}=0^{\circ}$, but $\hat{\gamma}+\partial^{2} \hat{\gamma} / \partial \phi^{2}$ in (2.19) attains its minimum there. The anisotropic profiles at this figure are plotted at $t=0,4,8,9,10,11,12$ and isotropic profiles are plotted at $t=0,4,8,12$ (same is true for Fig. 6(b)). In the anisotropic case, we see the formation of the spike near the corner of the mask and a very rapid lateral overgrowth on the mask. The spike does not change it's location in the horizontal direction and the overall vertical growth is slow. The overgrowth on the mask is almost completed by $t_{f}=12$.

Almost the same behaviour is observed in the six-fold case, Fig. 6(b). Here we chose $\epsilon_{\gamma}=1 / 50$. The minimum of $\hat{\gamma}$ is in the direction normal to the substrate (at $\phi=90^{\circ}$ ) when $\beta_{\gamma}=0^{\circ}$, but $\hat{\gamma}+\partial^{2} \hat{\gamma} / \partial \phi^{2}$ in (2.19) attains its maximum there. The only difference between Figures 6(a) and 6(b) is that the spike in Fig. 6(b) is less pronounced.

However, the behaviour changes drastically in both cases if we allow nonzero values of $\beta_{\gamma}$ (Fig. 6(c)). The choice of $\beta_{\gamma}=45^{\circ}$ in the four-fold case replaces 'minimum' by 'maximum' and vice versa in the discussion of Fig. 6(a) above, same true for the six-fold case if $\beta_{\gamma}=30^{\circ}$. The surface in Fig. 6(c) is smooth and the influence of the anisotropy is neglible.

We don't have an immediate explanation of Fig. 6. It should be noted that the normal velocity (2.19) is very complicated in $\hat{\gamma}+\partial^{2} \hat{\gamma} / \partial \phi^{2}$ and therefore it is unclear how to connect values of $\hat{\gamma}+\partial^{2} \hat{\gamma} / \partial \phi^{2}$, or $\hat{\gamma}$, along the curve at different values of $\beta_{\gamma}$ with values of $V_{n}$ along the curve. It is clear, however, that zero phase shifts result in large $V_{n}$ in the lateral direction (at $\phi=180^{\circ}$ ) and in small $V_{n}$ in the direction normal to the substrate, at $\phi=90^{\circ}$. Also, the results presented in the two preceeding sections suggest that (at least for the values of the nondimensional parameters used in this paper, ref. Table 2) the crystal growth is mainly controlled by the mobility; essentially by the impinging flux of particles from vapor, ref. (2.7), (2.10) (or (2.19)). And, the surface diffusion is not strong enough to smooth completely, in the short time the pronounced irregularities develop on the surface, if they do 

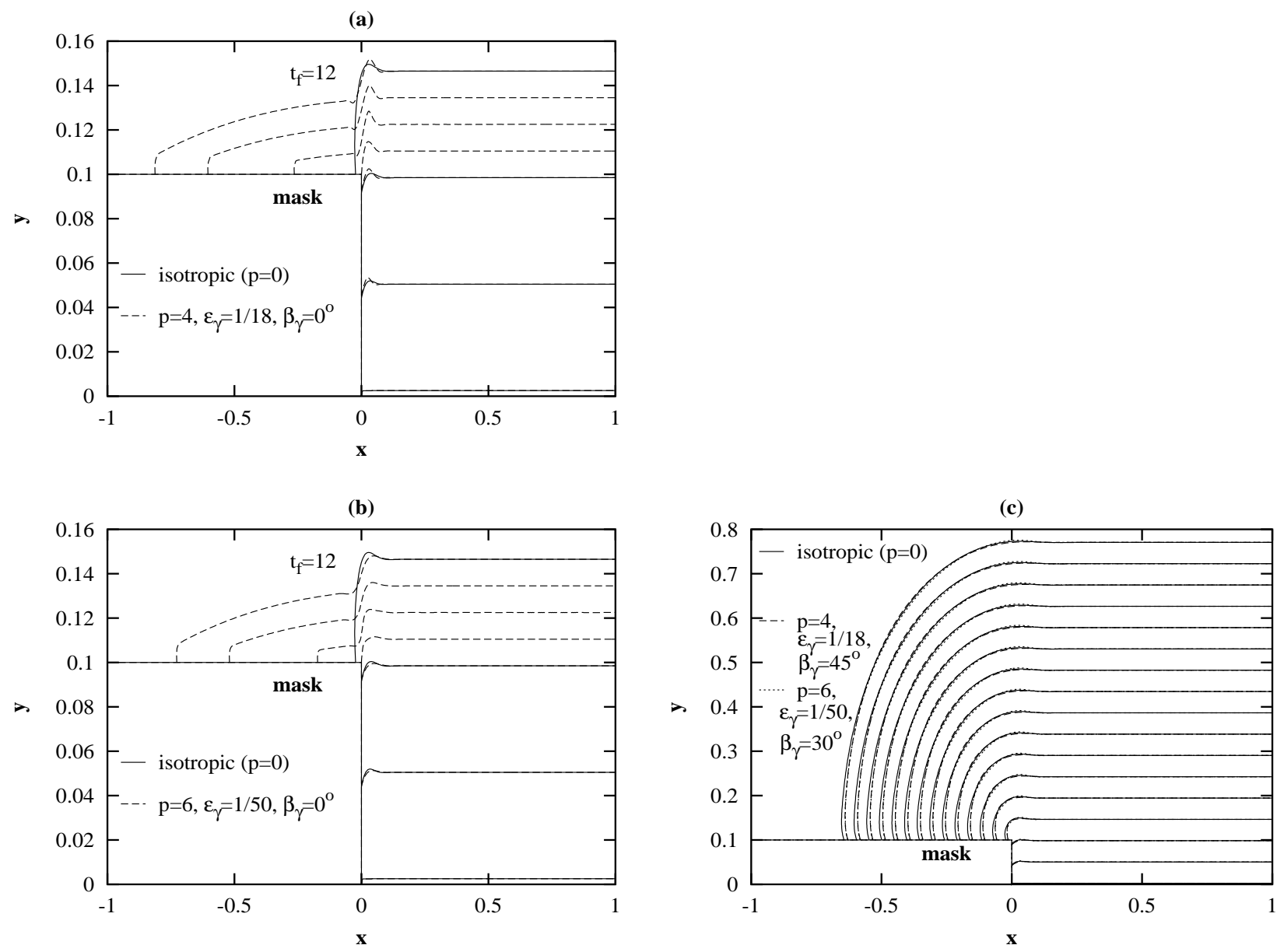

Figure 6: Comparison of anisotropic crystal growth to isotropic one; the growth anisotropy is due only to the anisotropy of the surface energy, $\gamma ; \epsilon_{m}=\epsilon_{d}=0$. (a) Four-fold anisotropy $(p=4), \beta_{\gamma}=0^{\circ}$. (b) Six-fold anisotropy $(p=6), \beta_{\gamma}=0^{\circ}$. (c) Same as (a), (b) but $\beta_{\gamma}=45^{\circ}$ in the four-fold case and $\beta_{\gamma}=30^{\circ}$ in the six-fold case.

occur (as in the case of Figures 6(a,b)). Indeed, we plotted the three terms that constitute the expression for the normal velocity (2.19) vs. the arc length and it appeared that the mobility term everywhere on the curve is one order of magnitute higher than the surface diffusion and the curvature terms. Reducing the value of the isotropic mobility, with all other parameters fixed, by up to a factor of 8 does not significantly change the results; reduction by a factor of 10 causes obviously incorrect results in the vicinity of the contact line. At this time, we believe that these combined effects are responsible for very strong overgrowth onto the mask and "freezing" of the spike.

\subsection{Anisotropic mobility, surface diffusion and surface energy $\left(\epsilon_{m} \neq 0, \epsilon_{d} \neq 0, \epsilon_{\gamma} \neq 0\right)$}

The purpose of this section is to provide a glance on how the anisotropic $M, D_{s}^{(c)}$ and $\gamma$ can act together. Since the crystal growth is controlled by the anisotropic mobility, we chose 
a relatively simple case presented in Fig. 2(b) (e.g., the "block" structure) to show the influence of anisotropic $D_{s}^{(c)}$ and $\gamma$. In this section, $p=4$.

In Fig. 7(a), we plot together the profiles corresponding to a very weak degree of anisotropy of the mobility, $\epsilon_{m}=1 / 18 . \epsilon_{\gamma}=1 / 18$ and $\epsilon_{d}=0.85$ for the solid curves, while $\epsilon_{\gamma}=\epsilon_{d}=0$ for the dashed curves. Note that we impose the phase shift $\beta_{\gamma}=45^{\circ}$, to avoid the irregular growth of the type shown in Figures $6(\mathrm{a}, \mathrm{b})$. As one could expect in this case, the crystal surface profiles are smooth, they closely resemble the isotropic surface profiles (ref. Fig. 5(a), for example). The difference between the growth with anisotropic $M$ only and the growth with anisotropic $M, D_{s}^{(c)}$ and $\gamma$ is small.

The increase of $\epsilon_{m}$ to 0.1 (having all other parameters fixed, Fig. 7(b)) results in the enhanced growth in the $\phi=135^{\circ}$ direction and formation of the corner. This corner is more pronounced for the solid curves due to the anisotropy of $\gamma$.

Finally, Fig. 7(c) shows the profiles for $\epsilon_{m}=0.25$. We see that the difference between the two modes of the growth increased; the two sets of curves are completely separated near the corner. The growth is faster for the case with $\epsilon_{m} \neq 0$ only.

We predict that unless $\beta_{\gamma}=0^{\circ}$, the fully anisotropic crystal shapes will be similar to the shapes presented in Figures $2-4 . \beta_{\gamma}=0^{\circ}$ results in a very different, irregular shapes of the type presented in Figures 6(a,b). In $p=4$ case, we obtained these shapes for $\epsilon_{\gamma}=1 / 36$. Unfortunately, our computation becomes unstable for larger values of $\epsilon_{\gamma}$ and we were unable to obtain the corresponding profiles. The increase of the grid resolution may help in this situation but the computation on a very fine grid $(N>1200-1500)$ is beyond the capabilities of the hardware currently at our disposal.

\section{Discussion and conclusions}

In this paper, the anisotropic growth from a trench in a masked substrate is computed. The previous isotropic model gave a small enhancement near the contact point; those bumps were small and were smoothed by surface diffusion; the larger the contact angle, the longer the smoothing took during the evolution. In the anisotropic case, much more spectacular enhancement of the growth near the contact point could be observed, or the growth could be quite similar to the isotropic case. The enhancement or lack of it depends on the anisotropy of the surface properties. It was demonstrated that, for a GaAs-like material, the anisotropy of the mobility of the crystal surface plays a crucial role in determining the shape of the crystal.

Markedly enhanced growth resulting in bumps near the contact line was typically caused when the fast growth directions were at intermediate angles with respect to the substrate (Figures 2(b), 4(b)). For the case of sixfold symmetry, the fast growth directions would be at $60^{\circ}, 120^{\circ}$ and $180^{\circ}$, while the direction normal to the substrate would be slow-growing, in order to see significantly enhanced growth near the contact point.

For strong surface diffusion and mobility anisotropy, the lateral overgrowth can be enhanced or suppressed very strongly simply by orienting the properties with respect to the substrate; such results were seen in Figure 3(b,d). When the fast growth direction is normal to the substrate, lateral overgrowth is essentially eliminated; when the slow growth direction is normal to the substrate, the overgrowth is quite large compared to the growth in the 

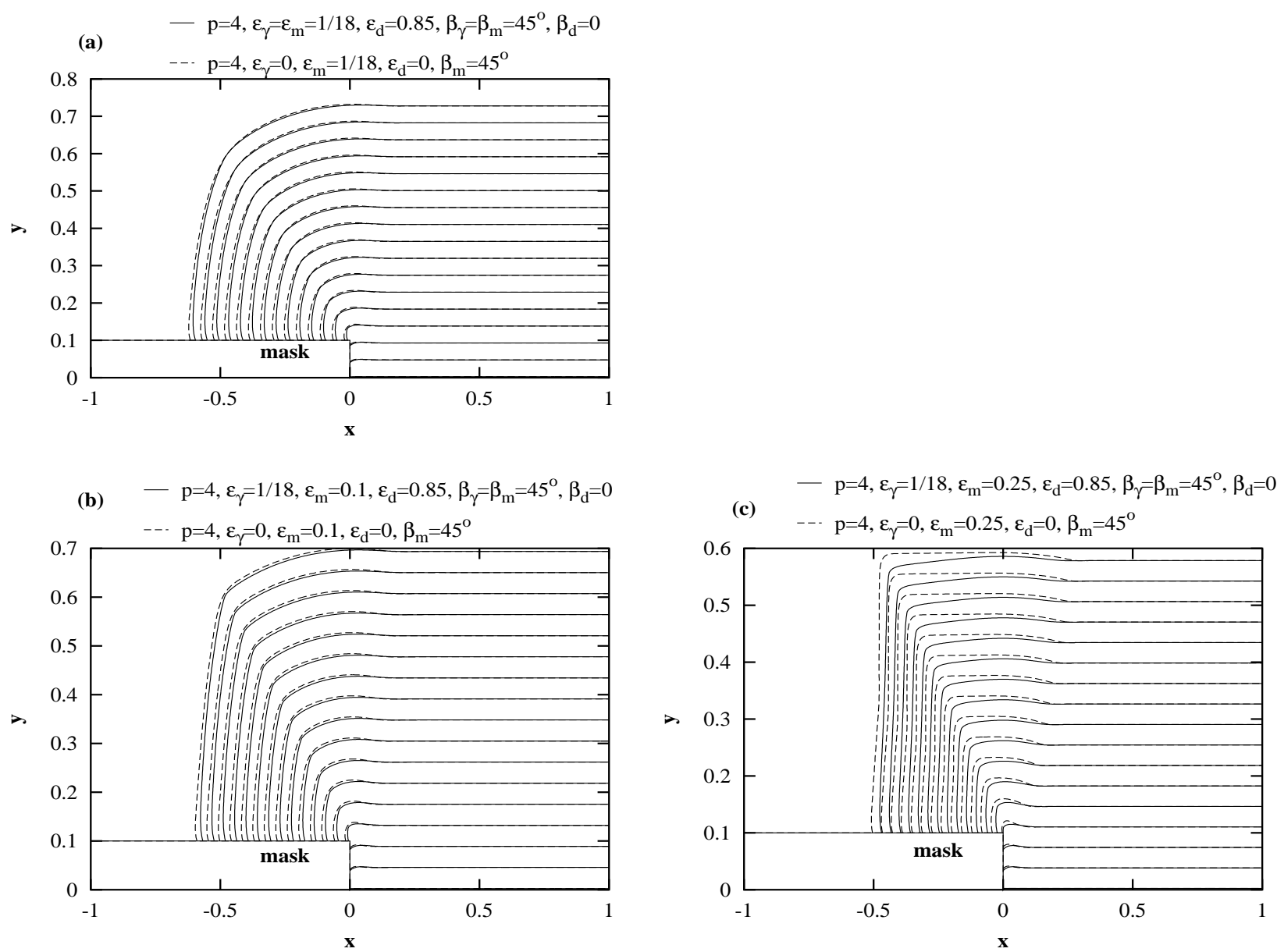

Figure 7: Comparison of the crystal growth with only $M$ anisotropic, to the fully anisotropic growth. $p=4$. (a): $\epsilon_{m}=1 / 18$. (b): $\epsilon_{m}=0.1$. (c): $\epsilon_{m}=0.25$.

center of the trench. Our model offers a way to quantify the effect of orienting the growing crystal compared to the substrate, and thus to possibly understand how to control the shape of the growing crystal.

The variety of shapes that can be produced from the model mimic a wide variety of observed growth. For example, a variety of block-like and truncated-pyramid shapes were observed (Figure 2(a,b,d) and Figure 3(a,d)) that are reminiscent of those observed experimentally by Nam et al. [8] with varying temperature (see Figure 4 in their paper). Our simulation assumes the geometrical parameters (mask thickness and windows width and separation) roughly equal to the parameters in their experiments. In [8], the amount of the crystal overgrowth on the mask as a function of growth time is not specified, but it is stated that "the amount of lateral growth exhibited a strong dependence on stripe orientation" (e.g., on the orientation of the growth with respect to the crystallographic axis of the substrate, the anisotropy captured by our model). They also observed increase of the growth rate of the crystals in both the lateral and the vertical directions (and also changes in shapes) with the increase of the supply of the material from vapor (see Figure 5 in [8]), similar to our computations with different mobility $M$. Also, we computed shapes similar 
to those found by Thrush et al. [6] (compare our Figures 2(c), 4(a,b) with Figures 3,4,5 in $[6])$.

There are some caveats about these results. One is that the level of anisotropy is almost certainly lower in our computations than in the experiment; a second is that we do not incorporate the temperature dependence in our model. The model takes the surface properties as input; accurate values for these parameters and the anisotropies are difficult to find. However, it is encouraging that with a sufficiently strong anisotropy and varying it can produce similar shapes. It is important to point out that in [6] the authors experimented with selective epitaxy of $\operatorname{InP} / \operatorname{GaInAs}(\mathrm{P})$, while in [8] the experiment was conducted with GaN. This leads us to speculate that the crystal structures such as those presented in Fig. 2 seem generic for the ELOG of III-V semiconductor materials. Our results could, at least partially, explain the overgrowth [6]-[9] or lack of it $[2,4]$ in the ELOG experiments.

The model could be extended by adding the computation of field variables on either side of the interface and their effect on the growth of the crystal. For example, the elastic stress in the crystal in growth from the vapor and the electric field in electroepitaxy from an alloy melt would be worthwhile extensions to the model. We are currently working on the latter.

\section{Acknowledgements}

M. Khenner and R.J. Braun were supported by NSF Grant DMS-9631287 and DMS-9722854. M.G. Mauk wishes to acknowledge support of the Ballistic Missile Defense Organization under Small Business Innovation Research contract DASG60-92-C-004.

\section{A Solution of full diffusion problem on the mask}

To account for the diffusion on vertical part of the mask, we first map two surfaces on the mask, see Fig. 1, to the single interval $I^{\prime}=\left\{X^{\prime}:\left[-\ell, h_{m}\right]\right\}$. The assumption underlying this approximation is that the mask corner has no effect on the diffusion of adatoms on the mask surface. (Note that the growing crystal is affected by the mask corner, however [18].) The diffusion equation (2.1) on the mask then reads

$$
\frac{\partial n_{m}}{\partial t^{\prime}}=D_{s}^{(m)} \frac{\partial^{2} n_{m}}{\partial\left(X^{\prime}\right)^{2}}+J_{g}-\frac{n_{m}}{\tau_{m}}, \quad-\ell \leq X^{\prime} \leq X_{*}^{\prime}\left(t^{\prime}\right), X_{*}^{\prime}\left(t^{\prime}\right) \in I^{\prime} .
$$

The nondimensional form of (A.1) is

$$
\frac{\partial u}{\partial t}=\frac{\partial^{2} u}{\partial X^{2}}+\alpha(1-u), \quad-d \leq X \leq X_{*}(t), X_{*}(t) \in I
$$

where $u$ is the nondimensional concentration, $\alpha$ is the material parameter (ref. Table 2) and $I=\left[-d, \bar{h}_{m}\right]$. The change of variables

$$
\xi=d \frac{X-X_{*}(t)}{d+X_{*}(t)}, \quad u(X, t)=U(\xi(t), t)
$$


transforms the equation (A.2) to

$$
\frac{\partial U}{\partial t}=\left(\frac{d}{d_{1}}\right)^{2} \frac{\partial^{2} U}{\partial \xi^{2}}+\dot{X}_{*}\left(\frac{d+\xi}{d_{1}}\right) \frac{\partial U}{\partial \xi}+\alpha(1-U), \quad-d \leq \xi \leq 0 .
$$

Here $\dot{X}_{*}$ denotes the time derivative of $X_{*}$ and

$$
d_{1}=d+X_{*}(t)
$$

is the length of the diffusion path on the mask, from the center of the mask $(X=-d)$ to the contact point $X_{*}(t)$. Note that the equation (A.4) is posed on the fixed interval of $\xi$. Appended to (A.4) are the boundary conditions

$$
\left.\frac{\partial U}{\partial \xi}\right|_{(-d, t)}=0, \quad U(0, t)=0
$$

and the initial condition

$$
U(\xi, 0)=0 .
$$

The initial-boundary value problem (A.4)-(A.7) is discretized in $\xi$ using central, second order finite differences; one-sided finite differences are used at $\xi=0$ to compute derivatives of $U$ there. 100 to 200 grid points are used for this discretization. The resulting set of coupled ordinary differential equations in time is integrated by the off-the-shelf solver RADAU [31]. To study the effects due to non-steady-state surface diffusion on the mask, we incorporated this integration into the overall computational cycle, so that the advancement of the crystal surface one time step forward is preceeded by the solution step of the problem (A.4)-(A.7) and the computation of the flux $f_{m}^{(\text {full })}$ from mask onto the cystal,

$$
f_{m}^{(\text {full })}=\left.\left(\frac{-d}{d_{1}}\right) \frac{\partial U}{\partial \xi}\right|_{(0, t)}
$$


(a)

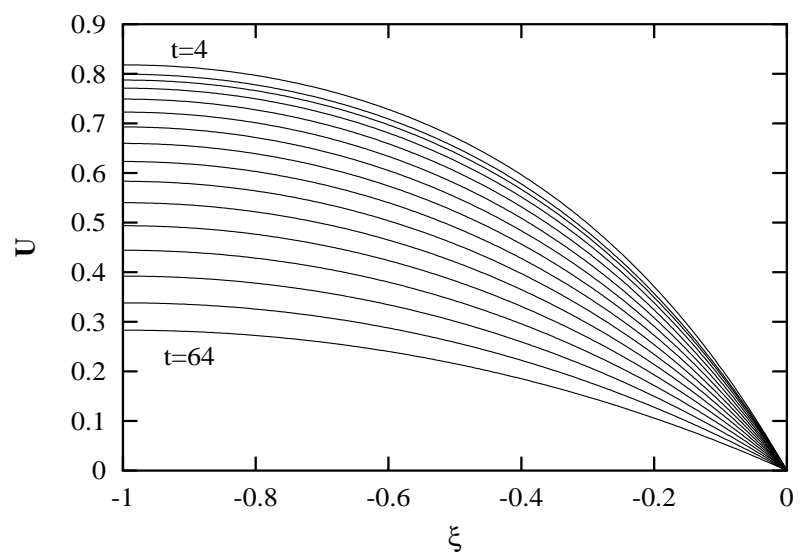

(b)

(c)
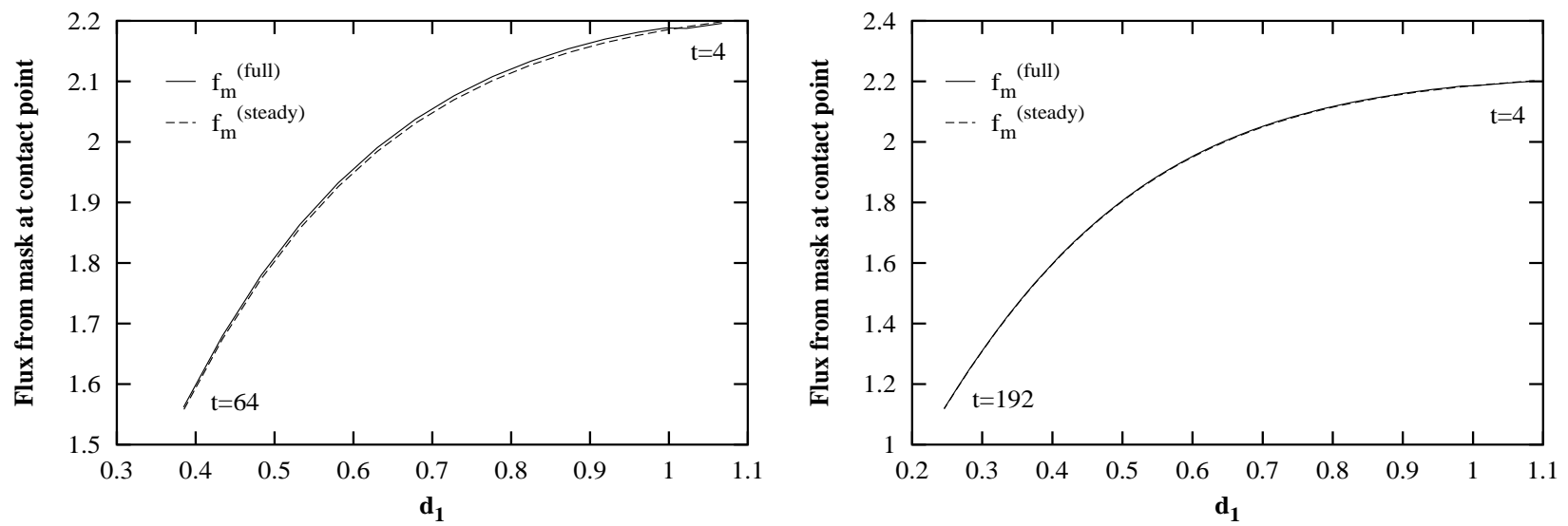

Figure 8: (a) Computed concentration profiles on the mask for the case of the isotropic ELOG. (b) The corresponding flux $f_{m}^{(\text {full) }}$ and the steady-state flux $f_{m}^{\text {(steady) }}$ at the contact point vs. the length of the diffusion path on the mask. (c) Same as (b), but for the anisotropic ELOG with $p=6, \epsilon_{m}=0.95, \epsilon_{d}=\epsilon_{\gamma}=0, \beta_{m}=0^{\circ}$.

Fig. 8 shows the concentration profiles $U(\xi)$ on the mask and $f_{m}^{(f u l l)} v s . d_{1}$. Also shown in Fig. 8(b) is the flux

$$
f_{m}^{(\text {steady })}=\sqrt{\alpha} \tanh \left(d_{1} \sqrt{\alpha}\right),
$$

which emerges as the steady-state solution of the boundary-value problem

$$
\frac{\partial^{2} u}{\partial X^{2}}-\alpha u=-\alpha,\left.\quad \frac{\partial u}{\partial X}\right|_{(-d)}=0, \quad u\left(X_{*}\right)=0 .
$$

Figures $8(\mathrm{~b}, \mathrm{c})$ clearly suggest that quasi-steady flux given by $f_{m}^{(\text {steady) }}$ is a good approximation to the full diffusion problem on the mask.

If we compare the numerical results using the constant flux approximation $f_{m}$, obtained by fixing the contact point at the mask corner and ignoring the diffusion along the vertical sidewall of the mask (ref. page 9 and [18]), with the results from the fully dynamic flux 
$f_{m}^{(\text {full })}$, the comparison is very good for the parameters used in our computations. This can be clearly seen in Fig. 9 and this result justifies the use of a constant flux at the contact point (crystal edge) in this paper and our previous work [18]. (Note that the mask surface is not shown in full in Fig. 9).

(a)

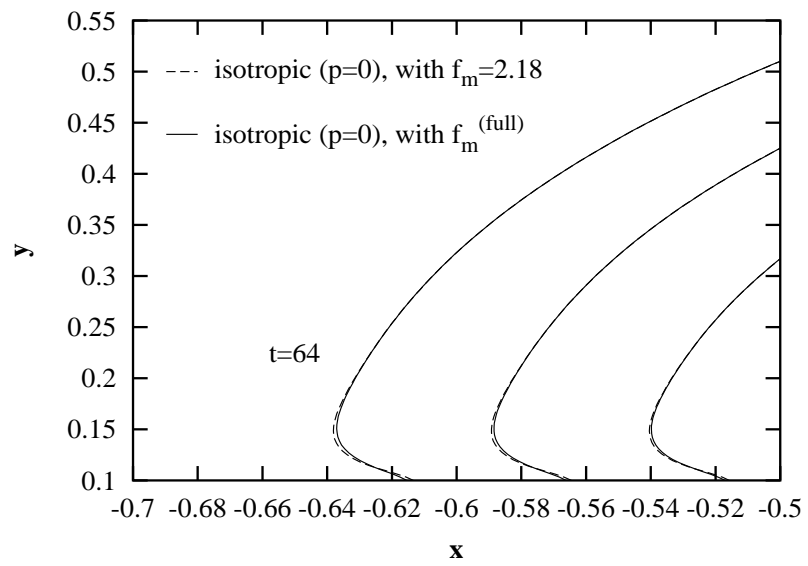

(b)

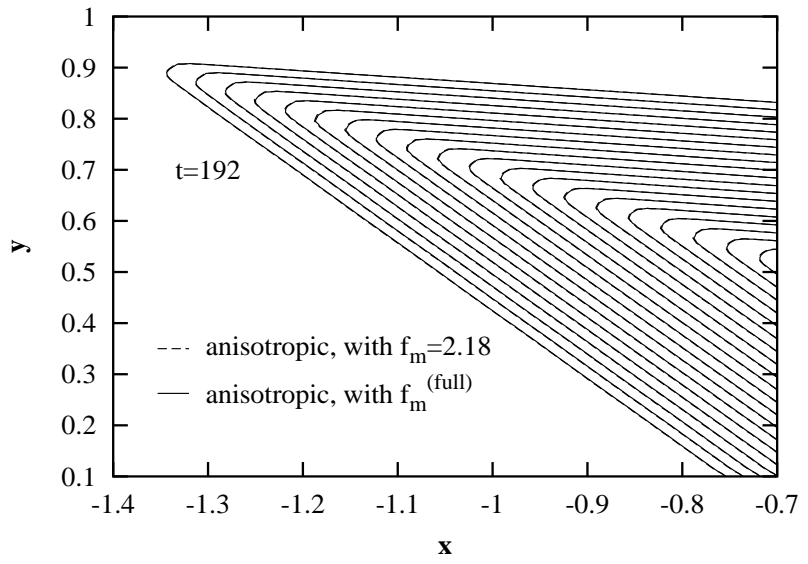

Figure 9: (a) Comparison of the isotropic crystal surface profiles computed with $f_{m}^{\text {(full) }}$ (see Fig. 8(b)) and with constant flux $f_{m}=2.18$; only the last three profiles are shown. (b) Same as (a), but for the anisotropic crystal growth with $p=6, \epsilon_{m}=0.95, \epsilon_{d}=\epsilon_{\gamma}=0, \beta_{m}=0^{\circ}$; here we show the zoom (at late time) into the region near the corner on the crystal surface in Fig. 3(c). The flux $f_{m}^{(\text {full })}$ is shown in Fig. 8(c).

\section{References}

[1] C.C. Mitchell, M.E. Coltrin, J. Han, J. Cryst. Growth 222 (2001) 144.

[2] T. Sasaki, M. Kitamura, I. Mito, J. Cryst. Growth 132 (1993) 435.

[3] Y. Sakata, Y. Inomoto, K. Komatsu, J. Cryst. Growth 208 (2000) 130.

[4] J.E. Greenspan, X. Zhang, N. Puetz, B. Emmerstorfer, J. Vac. Sci. Technol. A 18(2) (2000) 648.

[5] T. Fujii, M. Ekawa, S. Yamazaki, J. Cryst. Growth 146 (1995) 475.

[6] E.J. Thrush, J.P. Stagg, M.A. Gibbon, R.E. Mallard, B. Hamilton, J.M. Jowett, E.M. Allen, Mater. Sci. and Eng. B21 (1993) 130.

[7] M.A. Gibbon, J.P. Stagg, C.G. Cureton, E.J. Thrush, C.J. Jones, R.E. Mallard, R.E. Pritchard, N. Collis, A. Chew, Semicond. Sci. Technol. 8 (1993) 998. 
[8] O.-K. Nam, T.S. Zheleva, M.D. Bremser, R.F. Davis, J. Electron. Mater. 27(4) (1998) 233.

[9] P. Fini, H. Marchand, J.P. Robertson, S.P. DenBaars, U.K. Mishra, J.S. Speck, J. Cryst. Growth 209 (2000) 581.

[10] D.A. Kessler, J. Koplik, H. Levine, Adv. Phys. 37 (1988) 255.

[11] J.E. Taylor, J.W. Cahn, C.A. Handwerker, J. Cryst. Growth 40 (1992) 1443.

[12] A.A. Golovin, S.H. Davis, A.A. Nepomnyaschy, Phys. Rev. E 59 (1999) 803.

[13] T.P. Schulze, R.V. Kohn, Physica D 132 (2001) 520.

[14] A.-L. Barabási, H.E. Stanley, Fractal Concepts in Surface Growth, (Cambridge University Press, Cambridge, 1995).

[15] D. Adalsteinsson, J.A. Sethian, J. Comput. Phys. 120 (1995) 128.

[16] D. Adalsteinsson, J.A. Sethian, J. Comput. Phys. 122 (1995) 348.

[17] D. Adalsteinsson, J.A. Sethian, J. Comput. Phys. 138 (1997) 193.

[18] M. Khenner, R.J. Braun, M.G. Mauk, J. Cryst. Growth 235 (2002) 425.

[19] H. Wong, P.W. Voorhees, M.J. Miksis, S.H. Davis, Acta Mater. 48 (2000) 1719.

[20] T. Nishinaga, Handbook of Crystal Growth 3 (1994) 666 (ed. D.T.J. Hurle, Elsevier Science).

[21] A.A. Chernov, Handbook of Crystal Growth 3 (1994) 458 (ed. D.T.J. Hurle, Elsevier Science).

[22] C. Herring, Structure and Properties of Solid Surfaces 5 (1952) (ed. R. Gomer and C.S. Smith, The University of Chicago Press).

[23] W.W. Mullins, J. Appl. Phys. 28(3) (1957) 333.

[24] S.R. Coriell, D. Turnbull, Acta Metall. 30 (1982) 2135.

[25] L.V. Mikheev, A.A. Chernov, J. Cryst. Growth 112 (1991) 591.

[26] M. Muschol, D. Liu, H.Z. Cummins, Phys. Rev. A 46 (1992) 1038.

[27] H.J.W. Zandvliet, Rev. Mod. Phys. 72(2) (2000) 593.

[28] R. Gomer, Rep. Prog. Phys. 53 (1990) 917.

[29] T. Ala-Nissila, S.C. Ying, Prog. Surf. Sci. 39 (1992) 227.

[30] P.W. Voorhees, S.R. Coriell, G.B. McFadden, R.F. Sekerka, J. Cryst. Growth 67 (1984) 425.

[31] E. Hairer, G. Wanner, J. Comput. Appl. Math. 111 (1999) 93. 